\title{
ASSESSMENT OF THE SAFETY CLIMATE AT UNIVERSITY HOSPITALS IN THE SLOVAK REPUBLIC FROM THE NURSES` PERSPECTIVE
}

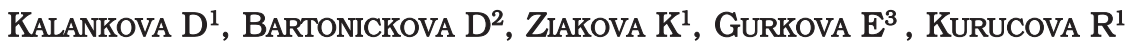 \\ ${ }^{1}$ Department of Nursing, Jessenius Faculty of Medicine in Martin, Comenius University in \\ Bratislava, Martin, Slovak Republic \\ ${ }^{2}$ Department of Nursing, 2nd Faculty of Medicine, Charles University in Prague, Czech Republic \\ ${ }^{3}$ Department of Nursing, Faculty of Health Sciences, Palacký University in Olomouc, \\ Czech Republic
}

\begin{abstract}
A b s tract
Introduction: Safety climate consists of individual dimensions that might be assessed using specific instruments, e.g., the Hospital Survey on Patient Safety Culture (HSOPS). Establishing the safety climate in healthcare facilities leads to improvements in patient safety.

Aim: To assess the safety climate at university hospitals in the Slovak Republic from the nurses' perspective and to determine the relationship between organisational variables and the particular components of the safety climate.

Methods: The study has a cross-sectional design. Data were collected using the HSOPS between December 2017 and July 2018. Two university hospitals participated in the study and overall 280 respondents were included. Respondents were recruited through the purposive sampling method. Data were analysed by descriptive and inductive statistics in the statistical programme SPSS 25.0.

Results: Results indicate that in the university hospitals there is a low-level of safety climate. The significant relationship was proved between organisational variables such as the experience in the current position, leaving intention, overtime, perception of staff adequacy, unit type, nurse-patient ratio, and the particular components of the safety climate.

Conclusion: Our findings may help hospital management to raise the awareness of the safety climate and to gain a sophisticated overview of the particular components of the safety climate. Adding new organisational variables may help to assess the safety climate from multiple perspectives and, thus, identify areas contributing to patient safety.
\end{abstract}

Keywords: Hospital Survey on Patient Safety Culture; nurses; assessment; patient safety; safety climate

\section{INTRODUCTION}

Patient safety is a crucial aspect of the provision of health care. Recently, patient safety has become a global challenge for all healthcare facilities, mainly because it is the central indicator of the quality of provided care $(1,2)$. The Institute of Medicine (IOM) describes safe care as the prevention of harm to patients caused by health care (3). Still, it also involves achieving the credible system of care as well as minimalising the incidence and the impact of the adverse events (4). It was proved that one of the strategies on how to improve the patient safety, reduce the adverse events, as well as to improve the quality of provided care is the development of the safety climate and its assessment in healthcare facilities (2). In previous years, the safety climate has been described as a broad, comprehensive, and multidimensional conceptual framework that is necessary for any organisation that strives to meet the safety goals by self-assessing the behaviour of healthcare professionals $(1,2)$. Based on the standard guidelines, the Hospital Survey on Patient Safety Culture (HSOPS)

Corresponding author: Mgr. Dominika Kalánková; e-mail: kalankova1@uniba.sk

(C) 2020 Dominika Kalankova et al.

This work is licensed under the Creative Commons Attribution-NonCommercial-NoDerivs 4.0 License (https://creativecommons.org/licenses/by-nc-nd/4.0/) 
instrument is commonly used in practice among the full range of questionnaires for monitoring the safety climate. The HSOPS is a valid and reliable instrument consisting of 42 items grouped into 12 dimensions. The instrument was translated into many languages and validated in different countries and different contexts (1). Although the tool is designed for the healthcare professional, nowadays, the literature points to the fact that the assessment of the safety climate should begin in the undergraduate studies $(5,6)$. In the Slovak Republic the safety climate was evaluated using the HSOPS in 2017 (7) with a sample of 1244 nurses. Since the nurses are the biggest group of healthcare professionals in hospitals included in the provision of nursing service, their assessment of the safety climate is necessary (8). The nature of their roles concerning the safety is an essential part of the provision of care (9) mainly because they are able to identify the risks to which the patients are exposed at an early stage of the hospitalisation (5). Evaluation of the safety climate from the nurses' perspective is essential mainly for the hospital management that may prevent all risks associated with patient safety through targeted interventions (1). Based on these facts, we would like to update the data to assess the safety climate at university hospitals in the Slovak Republic, which may contribute to the Slovak management in identifying threats associated with patient safety from the nurses' perspective as direct providers of nursing care. In addition, we would like to determine the relationship between the selected organisational variables and the particular components of the safety climate as the predicted relationship between those variables was confirmed in previously published studies $(10,11)$.

\section{METHODS}

The study has a cross-sectional design. The study was conducted between December 2017 and July 2018 and was approved by the Ethical committee of JFMED CU (30/2017). This is the sub-study of national research focusing on the safety climate in the Slovak Republic. Distribution of questionnaires was performed via head nurses in the selected hospitals in the Slovak Republic. Overall, three university hospitals were asked to participate in our research; two of them provided the consent with the conduction of the study.

\section{Sample}

Respondents were recruited by the purposive sampling method using the predefined criteria. Respondents were included in the research if they: a) worked as registered nurses (RNs), b) worked at medical-surgical, geriatric, and/or intensive care units, c) worked for at least one year. Respondents were not included if they were holding managerial positions.

\section{Data collection}

To measure the safety climate at university hospitals, the standardised questionnaire HSOPS released by AHRQ (12) was used. The questionnaire was designed for use by all healthcare staff. Still, for the purposes of our study, we distributed the questionnaires only to registered nurses as the largest group of healthcare staff providing direct care to patients. However, medical or nursing students, physicians, or other healthcare professionals might assess the safety climate in the original version, too. The body of HSOPS consists of nine parts (A-I). The central part of the instrument (A-D, F) reflects the 12 dimensions of the safety climate as well as two assigned items that were designed for evaluation of the overall perception of patient safety (E) and exploration of the number of adverse events reported during the last 12 months (G). The part $\mathrm{H}$ of the original version of the instrument includes six demographic data and the part I consists of the field in which respondents may comment on the safety climate. These two parts were not used in our study. Instead of the $\mathrm{H}$ part we used our own organisational variables (experience in the current position; experience in the nursing profession; leaving intention - current position, workplace, profession; overtime; perception of staff adequacy; nurse-patient ratio; unit type) which were selected based on the contem- 
porary literature and within the context of safety climate we consider very important. Dimensions of safety climate may be interpreted as the individual components/dimensions ('Teamwork within Units'; 'Supervisor/Manager Expectations and Actions Promoting Patient Safety'; 'Organisational Learning and Continuous Improvement'; 'Communication Openness'; 'Feedback and Communication about Error'; 'Nonpunitive Response to Errors'; 'Staffing'; 'Management Support for Patient Safety'; 'Teamwork across Units'; 'Handoffs and Transitions'; 'Overall Perception of Patient Safety'; and 'Frequency of Events Reported'), further reported in abbreviated form. Response options in the parts A-D in the questionnaire can be recorded using the 5-point Likert scale within the range from 1 - 'Strongly disagree', 2 - 'Disagree', 3 - 'Neither', 4 - 'Agree', 5 - 'Strongly agree'; and from 1 - 'Never', 2 - 'Rarely', 3 - 'Sometimes', 4 - 'Most of the time', 5 - 'Always'. The part E (Overall perception of patient safety) is assessed within the range from A (excellent) to $E$ (failing) and the part G (Frequency of events reported) comprise of 6 response options ( $\mathrm{a}$ - „no event reports “, $\mathrm{b}$ - „1 to 2 event reports“, c - „3 to 5 event reports“, d - „6 to 10 event reports“, e - „11 to 20 event reports “ to $\mathrm{f}-, 21$ event reports or more“). According to the AHRQ, parts A-D of the instrument should be scored by analysing the percentage of positive responses. Besides, in case the rate of positive responses is $75 \%$ or higher, we can consider the strong support of the safety climate (12). Parts $\mathrm{E}$ and $\mathrm{G}$ of the instrument should be scored by reporting the mean values.

\section{Data analysis}

Data were analysed in the statistical programme IBM SPPS Statistics 25.0 (IBM Corp., Armonk, New York, NY, USA). The data were proceeded through descriptive statistics (frequencies, M, SD). For the assessment of the relationship between the organisational variables and the particular components of the safety climate the statistical test One-way ANOVA was used. For the specific parts of the instrument as well as for the whole instrument the Cronbach alpha coefficient was calculated. The results were tested on the significance level $\mathrm{p} \leq 0.05$.

\section{RESULTS}

Overall, 446 questionnaires were distributed and 285 questionnaires were returned. Of these, five questionnaires were not completed and, therefore, were excluded, leaving a total of $280 \mathrm{RNs}$ (response rate -62.78\%). Sample characteristics are reported fully in Table 1.

\section{Assessment of the patient safety culture from the nurses perspective}

Evaluation of the safety climate from the nurses perspective at university hospitals across the Slovak Republic has revealed that RNs evaluated the safety climate under the expected level that is according to the AHRQ over $75 \%(10)$. The results are reported in detail in Table 2. The worst evaluated components were "Staffing", "Nonpunitive Responses", and "Teamwork across Units". The highest-assessed components were "Feedback" which was only slightly under the expected level, followed by "Organisational Learning" and "Supervisor Expectation". Evaluation of the nurse-reported patient safety grade and the number of events reported are illustrated in Table 3. Most RNs evaluated the overall grade of patient safety as very good, followed by acceptable. In addition, most of RNs addressed that they reported none adverse events within the last twelve months, followed by RNs reported between 3 to 5 adverse events within the previous twelve months.

\section{Relationship between the selected organizational variables and the components of the safe- ty climate}

The relationship between the selected organizational variables and the particular components of the safety climate was analyzed using ANOVA and is reported in Table 4. Mean values and standard deviations for the specific components of HSOPS concerning inde- 
Table 1 Sample characteristics

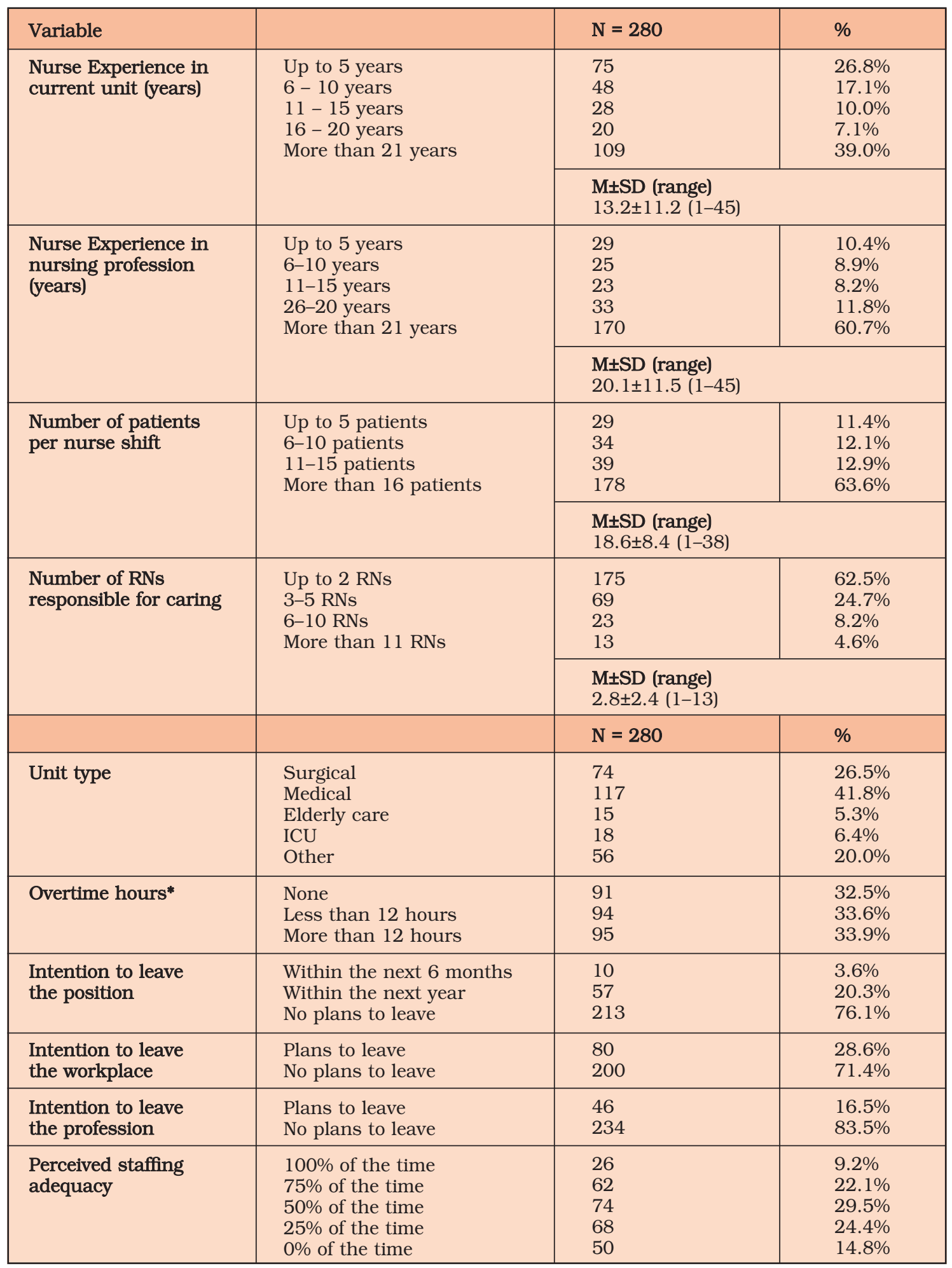

*in past three months 


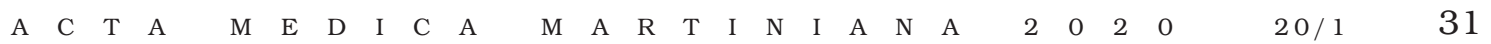

Table 2 Descriptive analysis of the particular components of the safety climate

\begin{tabular}{|l|c|}
\hline Components of the safety climate & The average percentage of positive responses \\
\hline Teamwork within Unit & $60.48 \%$ \\
\hline Supervisor Expectations & $65.73 \%$ \\
\hline Organisational Learning & $68.37 \%$ \\
\hline Management Support & $57.93 \%$ \\
\hline Perception Safety & $64.17 \%$ \\
\hline Feedback & $72.83 \%$ \\
\hline Communication Openness & $49.97 \%$ \\
\hline Teamwork across Units & $48.63 \%$ \\
\hline Staffing & $37.23 \%$ \\
\hline Handoffs & $55.05 \%$ \\
\hline Nonpunitive Responses & $41.83 \%$ \\
\hline Frequency of Events Reported & $63.70 \%$ \\
\hline
\end{tabular}

Table 3 Patient safety grade and events reported by nurses

\begin{tabular}{|l|c|c|}
\hline Patient safety grade & $\mathrm{n}=\mathbf{2 8 0}$ & \% \\
\hline Excellent & 22 & $49.1 \%$ \\
\hline Very good & 137 & $39.2 \%$ \\
\hline Acceptable & 110 & $3.4 \%$ \\
\hline Poor & 10 & $0.4 \%$ \\
\hline Failing & 1 & $\%$ \\
\hline Number of events reported & $\mathbf{n}=280$ & $37.8 \%$ \\
\hline No event reports & 106 & $21.5 \%$ \\
\hline 1 to 2 event reports & 60 & $23.0 \%$ \\
\hline 3 to 5 event repors & 64 & $12.9 \%$ \\
\hline 6 to 10 event reports & 36 & $3.8 \%$ \\
\hline 11 to 20 event reports & 11 & $1.0 \%$ \\
\hline 21 event reports or more & 3 & \\
\hline
\end{tabular}




\begin{tabular}{|c|c|c|c|c|c|c|c|c|c|c|c|c|c|}
\hline \multirow{11}{*}{ 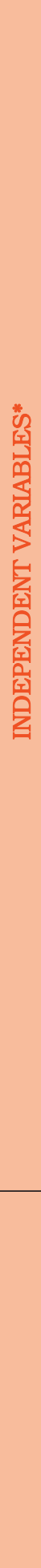 } & Кэеnbәре жетS & $\begin{array}{l}8 \\
8 \\
\end{array}$ & $\begin{array}{l}\text { ஜి } \\
\text { రి } \\
0\end{array}$ & $\begin{array}{l}\text { 옹 } \\
\text { N } \\
0\end{array}$ & $\begin{array}{l}\overrightarrow{\widehat{N}} \\
\text { ஸे }\end{array}$ & $\underset{\stackrel{n}{\stackrel{N}{*}}}{\stackrel{0}{0}}$ & $\stackrel{+}{\stackrel{+}{+}}$ & $\begin{array}{l}\overrightarrow{\text { }} \\
\text { ஸे }\end{array}$ & $\begin{array}{l}\vec{F} \\
\infty \\
\dot{0}\end{array}$ & $\begin{array}{l}8 \\
8 \\
\end{array}$ & $\begin{array}{l}\text { 요 } \\
8 \\
0\end{array}$ & $\begin{array}{l}\stackrel{+}{二} \\
\stackrel{0}{0}\end{array}$ & 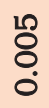 \\
\hline & 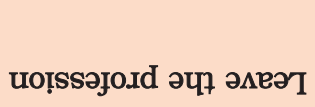 & $\begin{array}{l}\stackrel{+}{N} \\
\stackrel{0}{0}\end{array}$ & $\begin{array}{l}\infty \\
\stackrel{2}{\circ} \\
0 \\
\dot{0}\end{array}$ & $\begin{array}{l}\infty \\
\stackrel{\infty}{0} \\
\stackrel{0}{0}\end{array}$ & $\begin{array}{l}\text { ণิ } \\
\text { ઠ } \\
\text { ○े }\end{array}$ & 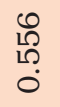 & $\begin{array}{l}\infty \\
\infty \\
+ \\
+\end{array}$ & $\begin{array}{l}\text { నै } \\
\stackrel{0}{0} \\
0\end{array}$ & $\begin{array}{l}0 \\
\infty \\
+ \\
0\end{array}$ & $\begin{array}{l}8 \\
0 \\
0 \\
0\end{array}$ & $\begin{array}{l}\text { ㅇ } \\
\text { ஸे } \\
\text { ஸे }\end{array}$ & 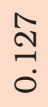 & $\begin{array}{l}8 \\
0 \\
0 \\
0\end{array}$ \\
\hline & 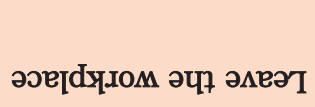 & $\begin{array}{l}8 \\
8 \\
\end{array}$ & $\begin{array}{l}\text { ஸి } \\
\text { ○ी } \\
0 \\
0\end{array}$ & $\begin{array}{l}\ddot{8} \\
\dot{0}\end{array}$ & $\begin{array}{l}8 \\
8 \\
0\end{array}$ & $\begin{array}{l}\text { ठ } \\
\\
\dot{0}\end{array}$ & $\begin{array}{l}\infty \\
\text { ơ } \\
\stackrel{0}{0}\end{array}$ & $\begin{array}{l}\text { ஸి } \\
\text { Оุ. } \\
0\end{array}$ & $\begin{array}{l}0 \\
\vdots \\
0 \\
0\end{array}$ & $\begin{array}{l}8 \\
8 \\
\end{array}$ & $\begin{array}{l}\tilde{8} \\
\dot{0}\end{array}$ & $\begin{array}{l}8 \\
8 \\
0\end{array}$ & $\stackrel{\infty}{\stackrel{\infty}{+}} \underset{0}{+}$ \\
\hline & 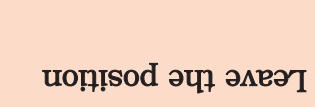 & 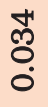 & $\begin{array}{l}\overrightarrow{0} \\
\stackrel{1}{0}\end{array}$ & $\begin{array}{l}\stackrel{N}{0} \\
\stackrel{1}{0}\end{array}$ & $\begin{array}{l}\text { 용 } \\
\text { రு }\end{array}$ & $\begin{array}{l}\bar{\theta} \\
\dot{0}\end{array}$ & $\begin{array}{l}\text { ชै } \\
0 \\
0\end{array}$ & $\begin{array}{l}\text { ठ̛ } \\
\text { ठ }\end{array}$ & $\begin{array}{l}10 \\
0 \\
0 \\
0\end{array}$ & $\begin{array}{l}8 \\
\text { ○ } \\
\text { ○े }\end{array}$ & 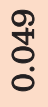 & \begin{tabular}{l}
+ \\
\multirow{0}{*}{}
\end{tabular} & $\begin{array}{l}\infty \\
0 \\
0 \\
0 \\
0\end{array}$ \\
\hline & 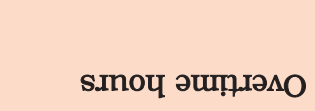 & $\begin{array}{l}\overrightarrow{0} \\
\stackrel{0}{0}\end{array}$ & $\begin{array}{l}\hat{0} \\
\text { 웅 } \\
\stackrel{0}{0}\end{array}$ & $\begin{array}{l}\infty \\
0 \\
0 \\
0\end{array}$ & $\begin{array}{l}\infty \\
\text { ஸి } \\
\text { ஸे } \\
0\end{array}$ & $\begin{array}{l}\stackrel{N}{+} \\
\stackrel{N}{0} \\
\stackrel{0}{*}\end{array}$ & $\begin{array}{l}\text { ه } \\
8 \\
0\end{array}$ & ஜ் & $\begin{array}{l}0 \\
10 \\
0 \\
0 \\
0\end{array}$ & $\begin{array}{l}8 \\
8 \\
\circ\end{array}$ & $\begin{array}{l}\overrightarrow{\mathrm{N}} \\
\text { ơ } \\
\text { o }\end{array}$ & $\begin{array}{l}\mathscr{8} \\
8 \\
0\end{array}$ & 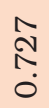 \\
\hline & SNY Jo ${ }^{\circ} \mathrm{N}$ & $\begin{array}{l}8 \\
\\
\end{array}$ & $\begin{array}{l}\vec{\infty} \\
\stackrel{\infty}{0} \\
\vdots\end{array}$ & $\begin{array}{l}8 \\
8 \\
0\end{array}$ & 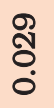 & $\underset{\overrightarrow{0}}{\overrightarrow{0}}$ & \begin{tabular}{l}
8 \\
8 \\
\hdashline
\end{tabular} & $\begin{array}{l}8 \\
8 \\
\end{array}$ & $\begin{array}{l}\mathscr{8} \\
80 \\
\dot{0}\end{array}$ & $\begin{array}{l}8 \\
8 \\
\end{array}$ & $\begin{array}{l}8 \\
8 \\
0\end{array}$ & $\begin{array}{l}8 \\
8 \\
0\end{array}$ & $\begin{array}{l}0 \\
\stackrel{1}{0} \\
0 \\
0\end{array}$ \\
\hline & squəฺ̣ed jo ${ }^{\circ} \mathrm{N}$ & $\begin{array}{l}\infty \\
0 \\
0 \\
0 \\
0 \\
0\end{array}$ & $\frac{\stackrel{10}{\oplus}}{\stackrel{\bullet}{0}}$ & $\begin{array}{l}\stackrel{0}{N} \\
\text { N̦ } \\
0\end{array}$ & 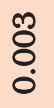 & $\begin{array}{l}\vec{\sigma} \\
\dot{0} \\
\dot{0}\end{array}$ & $\begin{array}{l}\check{8} \\
\dot{0}\end{array}$ & 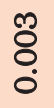 & $\begin{array}{l}m \\
\stackrel{n}{0} \\
0 \\
0\end{array}$ & $\begin{array}{l}\text { ஓे } \\
\text { ○ } \\
\text { ல }\end{array}$ & $\begin{array}{l}\text { 웜 } \\
\stackrel{0}{0}\end{array}$ & 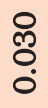 & $\begin{array}{l}\text { 尺े } \\
\text { లి } \\
0\end{array}$ \\
\hline & uo!̣ssəjo.dd ụ •dx' & $\stackrel{\infty}{\overparen{N}}$ & $\begin{array}{l}\hat{\infty} \\
+ \\
0\end{array}$ & $\begin{array}{l}\text { 尺े } \\
\text { లి } \\
0\end{array}$ & $\begin{array}{l}\text { ஸे } \\
\text { ஸे } \\
0\end{array}$ & $\begin{array}{l}\hat{0} \\
\stackrel{0}{0} \\
0\end{array}$ & $\begin{array}{l}\infty \\
\stackrel{\infty}{m} \\
\stackrel{0}{0}\end{array}$ & $\begin{array}{l}\text { மొ } \\
\stackrel{9}{+} \\
0\end{array}$ & $\begin{array}{l}\stackrel{+}{+} \\
\stackrel{0}{0}\end{array}$ & $\begin{array}{l}\overrightarrow{\hat{N}} \\
\text { oे } \\
0\end{array}$ & $\begin{array}{l}\stackrel{0}{+} \\
\stackrel{+}{0}\end{array}$ & 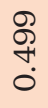 & $\begin{array}{l}\hat{N} \\
\stackrel{0}{0}\end{array}$ \\
\hline & 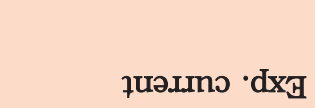 & $\begin{array}{l}0 \\
\infty \\
\oplus \\
0 \\
0\end{array}$ & $\begin{array}{l}\underset{H}{\not} \\
\stackrel{7}{0}\end{array}$ & $\begin{array}{l}\stackrel{+}{5} \\
\stackrel{5}{0} \\
\dot{0}\end{array}$ & $\begin{array}{l}\text { 우 } \\
\text { مُ } \\
\text { o }\end{array}$ & $\underset{\dot{O}}{\mathbb{Z}}$ & 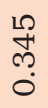 & $\begin{array}{l}\text { ஜి } \\
\text { ஸి } \\
\stackrel{0}{0}\end{array}$ & $\begin{array}{l}\text { ⿵⺆ } \\
\text { Lి } \\
0 \\
0\end{array}$ & $\begin{array}{l}\text { ஸे } \\
\text { ஸి } \\
\text { மे } \\
0\end{array}$ & $\stackrel{\stackrel{f}{\sharp}}{\stackrel{0}{\circ}}$ & $\begin{array}{l}\infty \\
\infty \\
\infty \\
0\end{array}$ & \begin{tabular}{l}
0 \\
\multirow{0}{0}{} \\
0
\end{tabular} \\
\hline & әdאุ $7 ! u_{\Lambda}$ & $\begin{array}{l}0 \\
\stackrel{0}{0} \\
\dot{0}\end{array}$ & $\begin{array}{l}\infty \\
\stackrel{2}{\circ} \\
0\end{array}$ & $\begin{array}{l}\overline{8} \\
0\end{array}$ & $\begin{array}{l}\text { ษ્ } \\
\text { ல }\end{array}$ & $\begin{array}{l}\infty \\
\infty \\
\circ \\
0\end{array}$ & $\begin{array}{l}0 \\
0 \\
0 \\
0\end{array}$ & $\begin{array}{l}\infty \\
\infty \\
\stackrel{0}{0}\end{array}$ & $\begin{array}{l}10 \\
\stackrel{0}{0} \\
\dot{0}\end{array}$ & $\begin{array}{l}\text { Nै } \\
\text { டे } \\
0\end{array}$ & $\begin{array}{l}8 \\
8 \\
0\end{array}$ & \begin{tabular}{l}
$\infty$ \\
\multirow{0}{*}{}
\end{tabular} & $\begin{array}{l}10 \\
\stackrel{1}{0} \\
0 \\
0\end{array}$ \\
\hline & 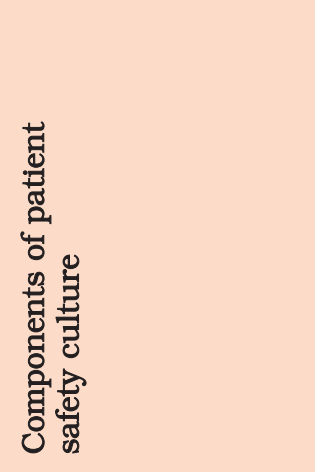 & 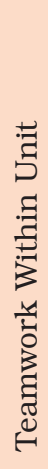 & 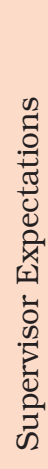 & 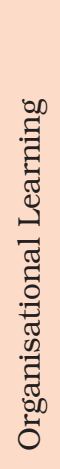 & 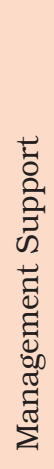 & 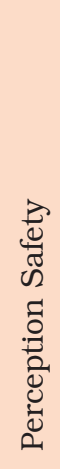 & 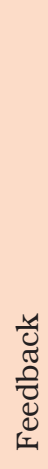 & 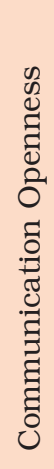 & 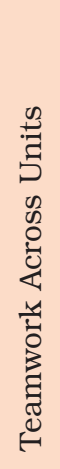 & 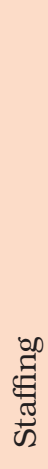 & 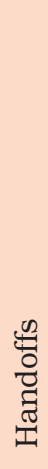 & 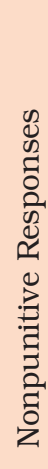 & 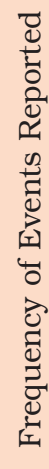 \\
\hline
\end{tabular}


pendent variables are not reported in Table 4, only the levels of statistical significance are reported.

There was not proved any significant relationship between the experience in the profession and the components of the safety climate. However, the significant association was confirmed between unit type and several components of safety climate. "Teamwork within Units" ( $\mathrm{M}=3.75, \mathrm{SD}=0.79)$, "Organisational Learning" $(\mathrm{M}=3.90, \mathrm{SD}=0.72)$, "Handoffs" $(\mathrm{M}=3.71, \mathrm{SD}=0.63)$, and "Nonpunitive Responses" $(\mathrm{M}=3.44, \mathrm{SD}=0.86)$ were the highest assessed components of safety climate by RNs working at medical care units. "Management Support" $(\mathrm{M}=3.47, \mathrm{SD}=0.65)$ was considered a strong dimension of safety climate by RNs working at surgical care units and "Feedback" $(\mathrm{M}=4.06, \mathrm{SD}=0.72)$ by $\mathrm{RNs}$ working at elderly care. Furthermore, "Teamwork within Units" was evaluated worst by RNs working at elderly care $(\mathrm{M}=3.16, \mathrm{SD}=0.94)$. Moreover, all other components of safety climate ("Organisational Learning" $(\mathrm{M}=3.31, \mathrm{SD}=0.64)$, "Management Support" $(\mathrm{M}=3.47, \mathrm{SD}=0.65)$, "Feedback" $(\mathrm{M}=3.35, \mathrm{SD}=0.61)$, "Handoffs" $(\mathrm{M}=3.19, \mathrm{SD}=0.59)$, and "Nonpunitive Responses" $(\mathrm{M}=3.13$, $\mathrm{SD}=0.65)$ ) were evaluated as lowest by RNs working at ICU.

A significant association was also found between the experience in the current unit and "Perception Safety". RNs who had been working at the current position for up to 5 years perceived their unit provided safe care to patients $(\mathrm{M}=3.75, \mathrm{SD}=0.71)$. On the contrary, $\mathrm{RNs}$ working at the current position for more than 21 years perceived the opposite $(\mathrm{M}=3.35$, $\mathrm{SD}=0.58)$. The trend shows the decreasing perception of providing safe care with the increasing experience at the current unit.

Furthermore, the perception of staff adequacy was significantly associated with the several components of the safety climate. When RNs perceived that the staffing was adequate at their shifts $(75 \%$ of the time), they evaluated "Teamwork within Units" $(\mathrm{M}=3.68, \mathrm{SD}=0.65)$, "Supervisor Expectation" $(\mathrm{M}=3.90, \mathrm{SD}=0.69)$, "Staffing" $(\mathrm{M}=3.36, \mathrm{SD}=0.77)$, "Handoffs" $(\mathrm{M}=3.75, \mathrm{SD}=0.57)$, and "Frequency of Events Reported" $(\mathrm{M}=4.10, \mathrm{SD}=0.76)$ better.

The number of patients of whom RNs was responsible to care of was significantly related to the several components of the safety climate. RNs who were responsible for number of patients between 11 and 15 considered "Management Support" (M=3.78, SD=0.56), "Feedback" (M=4.10, $\mathrm{SD}=0.64)$, "Communication Openness" $(\mathrm{M}=3.19, \mathrm{SD}=0.75)$, and "Nonpunitive Responses" $(\mathrm{M}=3.39, \mathrm{SD}=0.77)$ as the strongest dimensions of the safety climate. Furthermore, "Teamwork across Units" $(\mathrm{M}=3.20, \mathrm{SD}=0.49)$ and "Handoffs" $(\mathrm{M}=3.31$, $\mathrm{SD}=0.63$ ) were recognized as the highest assessed components by RNs who were responsible for caring of maximum to 5 patients. On the contrary, the worst evaluated components ("Management Support" (M=3.31, $\mathrm{SD}=0.39), \quad$ "Feedback" $(\mathrm{M}=3.44, \quad \mathrm{SD}=0.70)$, "Communication Openness" ( $\mathrm{M}=2.86, \mathrm{SD}=0.67)$, and "Nonpunitive Responses" $(\mathrm{M}=3.05$, $\mathrm{SD}=0.68)$ ) were by $\mathrm{RNs}$ responsible for caring of less than five patients. Still, components "Teamwork across Units" and "Handoffs" were evaluated lowest by RNs who cared of 6 to 10 patients per shift. It might be stated that RNs who were responsible for caring of fewer patients assessed the components mentioned above much better compared to those who were responsible for a higher number of patients.

Furthermore, the number of RNs responsible for caring was significantly associated with almost all of the safety climate components. "Teamwork within Units" $(\mathrm{M}=3.95, \mathrm{SD}=0.20)$, "Organisational Learning" $(\mathrm{M}=4.84, \mathrm{SD}=0.37), \quad$ Feedback" $(\mathrm{M}=4.49, \quad \mathrm{SD}=0.47)$, "Communication Openness" ( $\mathrm{M}=3.86, \mathrm{SD}=0.37)$, "Staffing” $(\mathrm{M}=4.10, \mathrm{SD}=0.39)$, "Handoffs" $(\mathrm{M}=4.42, \mathrm{SD}=0.21)$, and "Nonpunitive Responses" $(\mathrm{M}=4.56, \mathrm{SD}=0.51)$ were assessed the highest when 6 to $10 \mathrm{RNs}$ were responsible for caring of patients. When more than $11 \mathrm{RNs}$ were responsible for caring of patients, the best-evaluated components of the safety climate were "Management Support" $(\mathrm{M}=3.66, \mathrm{SD}=0.47)$ and "Teamwork across Units" $(\mathrm{M}=3.50$, $\mathrm{SD}=0.70$ ). On the contrary, almost all of those components ("Teamwork within Units" $(\mathrm{M}=3.37, \mathrm{SD}=0.77)$, "Organisational Learning” $(\mathrm{M}=3.67, \mathrm{SD}=0.60)$, "Feedback" $(\mathrm{M}=3.73$, $\mathrm{SD}=0.70)$, "Communication Openness" $(\mathrm{M}=3.14, \mathrm{SD}=0.77)$, "Staffing” $(\mathrm{M}=2.81, \mathrm{SD}=0.64)$, "Management Support" (M=3.48, $\mathrm{SD}=0.65)$, and "Teamwork across Units" (M=3.07, 
$\mathrm{SD}=0.36)$ ) were assessed worst by RNs when there were only 2 of them responsible for caring of patients. Logically, "Handoffs" $(\mathrm{M}=3.29, \mathrm{SD}=1.47)$ and "Nonpunitive Responses" $(\mathrm{M}=2.66, \mathrm{SD}=0.47)$ were evaluated worst when more than $11 \mathrm{RNs}$ were responsible for caring of patients.

Moreover, the number of overtime hours was significantly associated with six components. When RNs had none overtime hours, they evaluated better "Feedback" (M=4.09, SD=0.68) and "Communication Openness" $(\mathrm{M}=3.44, \mathrm{SD}=0.79)$. However, when they had between 1 and 12 overtime hours within the previous three months, they considered "Organisational Learning" ( $\mathrm{M}=3.92, \mathrm{SD}=0.71)$, "Staffing” $(\mathrm{M}=3.16, \mathrm{SD}=0.82)$, "Handoffs" $(\mathrm{M}=3.66, \mathrm{SD}=0.66)$, and "Nonpunitive Responses" $(\mathrm{M}=3.50, \mathrm{SD}=0.89)$ as the strongest dimensions of the safety climate. Likewise, all of the components mentioned above were perceived as the weakest by RNs who had more than 12 overtime hours within the past three months.

Besides, the intention to leave the current position was significantly related to several components. RNs who had no plans to leave the current position considered the following dimensions as the strongest ones: "Teamwork within Units" $(\mathrm{M}=3.47, \mathrm{SD}=0.76)$, "Management Support" (M=3.51, SD=0.58), "Communication Openness" (M=3.39, $\mathrm{SD}=0.79)$, "Staffing” $(\mathrm{M}=3.09, \mathrm{SD}=0.77)$, "Handoffs" $(\mathrm{M}=3.57, \mathrm{D}=0.62)$, and "Nonpunitive Responses" ( $\mathrm{M}=3.39, \mathrm{SD}=0.80)$, while those dimensions were concurrently evaluated as the worst by RNs who planned to leave the current position within the following six months.

Between intention to leave the workplace and almost all of the components of HSOPS a significant relationship was confirmed. RNS who did not plan to leave the workplace evaluated the following components better than RNs who had intentions to leave the workplace: "Teamwork within Unit" $(\mathrm{M}=3.50, \mathrm{SD}=0.74)$, "Organisational Learning" $(\mathrm{M}=3.84, \mathrm{SD}=0.71)$, "Management Support" (M=3.53, SD=0.57), "Perception Safety" (M=3.62, SD=0.61), "Feedback" (M=4.01, SD=0.71), "Staffing" $(M=3.10, \mathrm{SD}=2.65)$, "Handoffs" $(\mathrm{M}=3.59, \mathrm{SD}=0.66)$, and "Nonpunitive Responses" ( $\mathrm{M}=3.43, \mathrm{SD}=0.77)$.

Only one significant relationship was proved between the intention to leave the profession and "Management Support" $(M=3.50, S D=0.60)$. RNs who had no intentions to leave the profession evaluated the mentioned component better than RNs who planned to leave the profession $(\mathrm{M}=3.14, \mathrm{SD}=0.65)$.

RNs who had no plans to leave the position, workplace, or the profession evaluated the components of the safety climate mentioned above better. Based on the results of ANOVA, it is essential to improve the variables that influence the assessment of the safety climate. At the same time, most of them fall under the competence of hospital management.

\section{Reliability of the HSOPS instrument}

Reliability of the instrument was analysed using the Cronbach alpha coefficient which was calculated for the whole instrument. The result $(0.87)$ proved the very acceptable reliability of HSOPS. Cronbach alpha coefficient was also calculated for the particular components of the safety climate resulting in the acceptable value (according to the AHRQ, the acceptance level is > 0.6). The values were for "Teamwork within Units" (0.79), "Supervisor Expectations" (0.72), "Organizational Learning" (0.72), "Management Support" (0.75), "Perception Safety" (0.75), "Feedback" (0.72), "Communication Openness" (0.73), "Teamwork across Units” (0.77), "Staffing” (0.74), "Handoffs” (0.74), “Nonpunitive Responses” (0.74), and "Frequency of Events Reported” (0.77).

\section{DISCUSSION}

Nowadays, the safety climate has become the subject of interest of many healthcare facilities around the world. Revealing the safety climate and its components is the first step in the improvement of patient safety at healthcare facilities. Our study aimed to assess the 
safety climate at university hospitals in the Slovak Republic from the nurses perspective but also to determine the significant relationship between the selected organisational variables and the components of the safety climate.

Within the overall assessment of safety climate, the highest evaluated component in our study was "Feedback" which was supported in only one available study (10). Many authors (14-21) identified that "Teamwork within Units" is the most frequently rated as the highest evaluated component of the safety climate. Simultaneously, some findings of international studies indicate in results that "Feedback" tends to be the weakest component of the safety climate (e.g., 22, 23). The explanation for this may be that "Feedback" is related to the adverse events reporting, which is required by the law in the Slovak Republic comparing to other countries. On the contrary, the lowest evaluated dimension was "Staffing" which was supported by many international studies (e.g., 11, 13, 21, 23-24) regarding the fact that shortage of staff is the worldwide problem. RNs in our study reported the overall grade of patient safety as "very good" to "acceptable", which Bodur and Filiz (15), Farzi et al. (17), and $\mathrm{Wu}$ et al. (25) supported in their studies as well. Besides, most of RNs reported none adverse events within the previous twelve months. Our findings are consistent with several international studies $(14,15,26,27)$.

In our study, the significant relationship between the experience in the profession and the components of the safety climate was not verified. This finding is consistent with the study of Ammouri et al. (10) and Günes et al. (19). However, we explored the significant association between unit type and the components, such as "Teamwork within Units", "Organisational Learning", "Management Support", "Feedback", "Handoffs", and "Nonpunitive Responses", which is supported in the study of Chen and Li (16). We identified the significant relationship between the experience in the current units and the component "Perception Safety". The same result illustrates the study of Ammouri et al. (10). Furthermore, we explored the significant association between the perception of staff adequacy and "Teamwork within Units", "Supervisor Expectations", "Staffing", "Handoffs", and "Frequency of Events Reported". No available study examined this relationship, but as the shortage of staff is the global problem, it is clear that it may influence the particular components of patient safety worldwide. Likewise, the number of RNs responsible for caring significantly relies on almost all of the safety climate components, which is in line with previously published studies (e.g., 11,22). With this issue, it is closely related the number of patients of whom RNs was responsible to care of, which influenced significantly "Management Support", "Feedback", "Communication Openness", "Teamwork across Units", "Handoffs", and "Nonpunitive Responses". In addition, these associations were not explored in the international context but we recommend focusing on them more intently. One of the significant predictors of worse evaluated components of safety climate "Organisational Learning", "Feedback", "Communication Openness", "Staffing", "Handoffs", and "Nonpunitive Responses" - was the number of overtime hours, which is directly related to the shortage of staff. Besides, the significant relationship was confirmed between the intention to leave the current position and/or workplace and the several components of the safety climate. Plan to leave the profession was associated significantly only with the component "Management Support". However, none of these associations was explored in international or national studies. Our study revealed critical findings that should be considered further by the hospital management. Improvements may relate to the particular organisational variables of university hospitals in the Slovak Republic. We would, therefore, like to point out on using the organisational variables that are not included in the original version of the HSOPS and to provide many other interesting findings contributing to the overall improvement of safety climate.

Cronbach alpha coefficient of 0.87 indicates a very acceptable reliability of the HSOPS. Our findings are consistent with the study of Bartoníčková et al. (27) and similar, e.g. the study of Hammer et al. (28) or Nordin et al. (29). On the contrary, many international studies revealed a very weak value of the Cronbach alpha coefficient for particular dimensions of 
HSOPS (e.g., 30-32), which may be caused by the problems with the translation process or the understanding of the items of HSOPS. Overall, according to the value of Cronbach alpha coefficient, we determine that this instrument is reliable for use in the Slovak Republic.

\section{Limitation of the study}

The study has several limitations. The first limitation is that the instrument HSOPS was not developed for the targeted language and culture. Besides, construct validity was not tested. Another limitation is the inclusion of a non-randomized sample of registered nurses and the integration of only two university hospitals based on the written permissions.

\section{CONCLUSION}

Through this study, we wanted to introduce a reliable version of the HSOPS questionnaire for its use in the Slovak Republic. In the overall assessment of the safety climate by using this Slovak version we found that RNs working at university hospitals evaluated as the highest component "Feedback and Communication about Error". The worst evaluated component was "Staffing". The overall grade of patient safety reported by RNs was "very good" to "acceptable"; most of RNs reported none adverse events within the previous twelve months. Due to a limited number of socio-demographic data in the original version of the instrument we established new organisational variables. We found out that experience in the current unit, the perception of staff adequacy, number of patients of whom RNs was responsible to care of, overtime hours, but also leaving intentions are considerable and significant predictors influencing increase or decrease in the assessment of the particular component of the safety climate. When evaluating these variables concerning the safety climate, it is possible to provide much more opportunities for management to identify areas affecting the decrease in quality of provided care. As these were significant variables that considerably influence particular components, we recommend its implementation in further studies so the safety climate may be advanced as well as the quality of provided care may be increased.

\section{REFERENCES}

1. Behzadifar M, Behzadifar M, Jahanpanah F, Bragazzi NL. Patient safety culture assessment in Iran using the "Hospital survey on patient safety culture" tool: A systematic review and meta-analysis. Clin Epidemiol Glob Health 2019. [Article in press].

2. Cheng C, Yen AMF, Lee YH. Factors affecting patient safety culture among dental healthcare workers: A nationwide cross-sectional survey. J Dent Sci 2019;14:263-268.

3. Institute of Medicine (US) Committee on Data Standards for Patient Safety; Aspden P, Corrigan JM, Wolcott J, Erickson SM, editors. Patient safety: achieving a new standard for care. Washington (DC): National Academies Press (US); 2004.

4. Kirwan M, Riklikiene O, Gotlib J, Fuster P, Borta M. Regulation and current status of patient safety content in pre-registration nurse education in 27 countries: Findings from the Rationing Missed nursing care (RANCARE) COST Action project. Nurse Educ Practice 2019; 37:132-140.

5. Bressan V, Stevanin S, Bulfone G, Zanini A, Dante A, Palese A. Measuring patient safety knowledge and competences as perceived by nursing students: An Italian validation study. Nurse Educ Practice 2016;16(1):209-216.

6. Usher K, Woods C, Parmenter G, Hutchinson M, Mannix J, Power T, Chaboyer W, Latimer S, Mills J, Siegloff L, Jackson D.Self-reported confidence in patient safety knowledge among Australian undergraduate nursing students: A multi-site cross-sectional survey study. Int J Nurs Stud 2017;71:89-96.

7. Sováriová Soósová M, Zamboriová M, Murgová A. Hospital patient safety culture in Slovakia. Ošetrovatelstvo: teória, výskum, vzdelávanie 2017;1(1):12-19. 
8. Alshammari F, Pasay-an E, Alboliteeh M, Alshammari MH, Susanto T, Villareal S, Indonto MCL, Gonzales F. A survey of hospital healthcare professionals' perceptions toward patient safety culture in Saudi Arabia. IJANS 2019;11:100149.

9. Kong Ln, Zhu WF, He S, Chen SZ, Yang L, Qi L, Peng X. Attitudes towards patient safety culture among postgraduate nursing students in China: A cross-sectional study. Nurse Educ Practice 2019; 38:1-6.

10. Ammouri AA, Tailakh AK, Muliira JK, Geethakrishnan R, Al Kindi SN. Patient safety culture among nurses. Int Nurs Rev 2015;62(1):102-110.

11. El-Jardali F, Sheikh F, Garcia NA et al. Patient safety culture in a large teaching hospital in Riyadh: baseline assessment, comparative analysis and opportunities for improvement. BMC Health Serv Res 2014; 14(1):1-15.

12. AHRQ- The Agency for Healthcare Research and Quality. Hospital Survey on Patient Safety Culture [online]. 2015 [cited 2019 Sep 15]. Available on: https://www.ahrq.gov/sops/surveys/ hospital/index.html

13. Wang X, Liu K, You LM, Xiang JG, Hu HG, Zhang LF, Zheng J, Zhu XW. The relationship between patient safety culture and adverse events: A questionnaire survey. Int J Nurs Stud 2014;51(8):1114-1122.

14. Alqattan H, Cleland J, Morrison Z. An evaluation of patient safety culture in a secondary care setting in Kuwait. J Taibah Univ Med Sci 2018;13(3):272-280.

15. Bodur S, Filiz E. Validity and reliability of Turkish version of "Hospital Survey on Patient Safety Culture and perception of patient safety in public hospitals in Turkey. BMC Health Serv Res 2010;10:28.

16. Chen IC, Li HH. Measuring patient safety culture in Taiwan using the Hospital Survey on Patient Safety Culture (HSOPSC). BMC Health Serv Res 2010;10:152.

17. Farzi S, Moladoost A, Bahrami M, Farzi S, Etminani R. Patient safety culture in intensive care units from the perspective of nurses: A cross-sectional study. Iran J Nurs Midwifery Res 2017; 22(5):372-376.

18. Giai J, Boussat B, Occelli P, Gandon G, Seigneurin A, Michel P, Francois P. Hospital survey on patient safety culture (HSOPS): variability of scoring strategies. Int $\mathrm{J}$ Qual Health $\mathrm{C}$ 2017;29(5):685-692.

19. Güneş ÜY, Gürlek Ö, Sönmez M. A survey of the patient safety culture of hospital nurses in Turkey. Collegian 2016;23(2):225-232.

20. Hefner JL, Hilligoss B, Knupp A, Bournique J, Sullivan J, Adkins E, Moffatt-Bruce SD. Cultural transformation after implementation of crew resource management: Is it really possible? Am $\mathrm{J}$ Med Qual 2017;32(4):384-390.

21. Khater WA, Akhu-Zaheya LM, Al-Mahasneh SI, Khater R. Nurses' perceptions of patient safety culture in Jordanian hospitals. Int Nurs Rev 2015;62(1):82-91.

22. Agnew C, Flin R, Mearns K. Patient safety climate and worker safety behaviours in acute hospitals in Scotland. J Safety Res 2013;45:95-101.

23. Nie Y, Mao X, Cui H, He S, Li J, Zhang M. Hospital Survey on Patient Safety Culture in China. BMC Health Serv Res 2013;13:228.

24. Robida A. Hospital Survey on Patient Safety Culture in Slovenia: a psychometric evaluation. Int J Qual Health C 2013;25(4):469-475.

25. Wu Y, Fuhita S, Seto K, Ito S, Matsumoto K, Huang CC, Hasegawa T. The impact of nurse working hours on patient safety culture: a cross-national survey including Japan, the United States and Chinese Taiwan using the Hospital Survey on Patient Safety Culture. BMC Health Serv Res 2013;13:394.

26. Mikušová V, Rusnáková V. Vnímanie kultúry bezpečnosti pacientov v nemocniciach na Slovensku. In: Jihočeská konference nelékařských zdravotnických pracovníků. České Budějovice: Nemocnice České Budějovice; 2012. s. 46-51. (in Slovak)

27. Bartoníčková D, Kalánková D, Mikšová Z, Žiaková K, Mazalová L. Patient safety culture from a nursing point of view in a broader context. Kontakt 2019;21(2):121-127.

28. Hammer A, Ernstmann N, Ommen O, Wirtz M, Manser T, Pfeiffer Y, Pfaff H. Psychometric pro- 
perties of the Hospital Survey on Patient Safety Culture for hospital management (HSOPS_M). BMC Health Serv Res 2011;11:165.

29. Nordin A, Wilde-Larsson B, Nordström G, Theander K. Swedish Hospital Survey on Patient Safety Culture - psychometric properties and healthcare staff's perception. Open J Nurs 2013;3(8A):41-50.

30. Brajshori N, Behrens J. Translation, cultural adaption and validation of Hospital Survey on Patient Safety Culture in Kosovo. Open J Nurs 2016;6(6):483-490.

31. Brborović H, Brborović O, Brumen V, Pavleković G, Mustajbegović J. Are nurse presenteeism and patient safety culture associated: a cross-sectional study. Arch Ind Hyg 2014;65(2):149-156

32. Ocelli P, Quenon JL, Kret M, Domecq S, Delaperche F, Claverie O, Castets-Fontaine B, Amalberti R, Auroy Y, Parneix P, Michel P. Validation of the French version of the Hospital Survey on Patient Safety Culture questionnaire. Int J Qual Health C 2013;25(4):459-468.

\section{Acknowledgements}

Supported by the project COST Action CA 15208 Rationing Missed Nursing Care: An international and multidimensional problem.

Received: February, 24, 2020

Accepted: March, 6, 2020 